\title{
Diversidade de Chironomidae (Diptera) em dois córregos de baixa ordem na região central do Estado de São Paulo, através da coleta de exúvias de pupa
}

\author{
Tadeu Siqueira ${ }^{1,2} \&$ Susana Trivinho-Strixino ${ }^{2}$
}

\begin{abstract}
'Programa de Pós-Graduação em Ecologia e Recursos Naturais, Universidade Federal de São Carlos. tadeucapuzzo@yahoo.com.br ${ }^{2}$ Laboratório de Entomologia Aquática, Depto. de Hidrobiologia, UFSCar Rod. Washington Luiz Km 235, Cx. Postal 676, 13565-905, São Carlos-SP, Brasil.
\end{abstract}

\begin{abstract}
Diversity of Chironomidae (Diptera) in two low order streams in the central region of São Paulo State, by collection of pupal exuviae. We analyzed the diversity and richness of lotic Chironomidae by collecting pupal exuviae. Drift nets and hand nets were used in a $100 \mathrm{~m}$ section in each stream in the dry season (August/2001). We analyzed and identified 1264 pupal exuviae belonging to 61 taxa (36 taxa in Fazzari stream and 31 in Monjolinho river section). Both systems showed low faunistic similarity reflecting the different characteristics of the substrate, canopy and water of each stream. Probably the highest richness and the presence of mining taxa like Stenochironomus spp., Xenochironomus spp. and Endotribelos spp. reflect the presence of the riparian forest in Fazzari stream, which contribute to habitat heterogeneity and food resource to these groups. The collection of pupal exuviae proved to be very efficient for characterizing the biodiversity of low order lotic systems.
\end{abstract}

KEYWORDS. Aquatic insects; Chironomidae pupal exuviae; lotic systems.

RESUMO. Diversidade de Chironomidae (Diptera) em dois córregos de baixa ordem na região central do Estado de São Paulo, através da coleta de exúvias de pupa. Estimamos a diversidade e a riqueza de Chironomidae (Diptera) em dois diferentes córregos através da análise de exúvias de pupa. As coletas realizadas no período de estiagem (agosto/2001) foram feitas com auxílio de redes de deriva e de mão, em trechos de 100 metros de cada córrego. Foram analisadas e identificadas 1264 exúvias de 61 táxons (36 no Córrego do Fazzari e 31 no trecho do Rio do Monjolinho). Ambos os sistemas apresentaram baixa similaridade faunística refletindo as diferentes características do substrato, da cobertura vegetal e da água de cada sistema. Possivelmente a maior riqueza faunística e a ocorrência de táxons minadores, como Stenochironomus spp., Xenochironomus spp. e Endotribelos spp. refletem a presença da mata ciliar no córrego do Fazzari que resulta em maior heterogeneidade ambiental e maior disponibilidade de alimento para esses grupos. O método de coleta e análise de exúvias de pupa de Chironomidae mostrou-se eficiente para estudos de caracterização ambiental de levantamentos da biodiversidade de sistemas lóticos de baixa ordem.

PALAVRAS-CHAVE. Exúvias de pupa de Chironomidae; insetos aquáticos; sistemas lóticos.

Os programas de avaliação da qualidade da água, os estudos de caracterização de rios e córregos, além de outros estudos de cunho limnológico, têm utilizado as formas imaturas de macroinvertebrados aquáticos como ferramenta de análise (Humphrey, Bishop \& Brown 1990; Humphrey \& Dostine 1994). Entretanto, não há um consenso entre os pesquisadores sobre qual grupo taxonômico que representa melhor as características ambientais do corpo d'água ou que forneça informações mais confiáveis a respeito da saúde ambiental (Ruse 2000).

Os insetos da família Chironomidae (Diptera), são importantes componentes da comunidade bentônica de sistemas lóticos e lênticos, colonizando uma grande variedade de biótopos e vivendo sob as mais diversas condições ambientais (Pinder 1986). As larvas desta família de insetos aquáticos, que geralmente dominam numericamente a comunidade de macroinvertebrados, têm sido negligenciadas nos estudos de caracterização de sistemas aquáticos (Hardwick et al. 1995). Esse fato se deve à dificuldade de identificação, ao grande esforço amostral e de laboratório que tem de ser empreendido e à confusão gerada em comparações de estudos feitos com diferentes métodos de coleta (Wilson \& Bright 1973; Ruse 2000).
Estes e outros problemas estimularam a criação de um procedimento mais rápido e barato para caracterizar e monitorar sistemas aquáticos. Com a "Chironomid Pupal Exuviae Technique” (CPET), desenvolvida por Wilson \& Mcgill (1979), exúvias de pupas de Chironomidae podem ser coletadas e analisadas utilizando o mesmo método independente do tipo de corpo d'água. As exúvias das pupas, após a emergência dos adultos permanecem na superfície por no máximo 2 dias, podendo se dispersar por deriva a distâncias não maiores do que 100 metros ou permanecer acumuladas nas margens ou em qualquer obstáculo como troncos ou folhas (Wilson \& Bright 1973).

O valor da utilização desta técnica para o estudo da diversidade de espécies de Chironomidae e para monitorar e caracterizar córregos e rios já foi demonstrado na Europa por Mcgill et al. (1979), Wilson $(1979,1984)$ e Ruse (2000); na América do Norte por Coffman (1973) e por Coffman \& De La Rosa (1998); na Austrália por Hardwick et al. (1995) e na África por Lehmann (1981). No Brasil a única publicação sobre exúvias é a de Stur et al. (2000) que analisa a fauna de um córrego sujeito a forte variação hidrológica na região do Mato Grosso.

O método possibilita uma coleta e triagem mais fácil e mais 
Tabela I. Lista de táxons e participação relativa (\%) no Córrego do Fazzari e Rio do Monjolinho.

\begin{tabular}{|c|c|c|c|c|c|}
\hline Táxons & Rio Monjol. & Cor. Fazzari & Táxons & Rio Monjol. & Cor. Fazzari \\
\hline Tanypodinae & & & Chironomini & & \\
\hline Ablabesmyia sp. 1 & 3,33 & 3,02 & Beardius xylophilus Trivinho- & - & 1,65 \\
\hline Ablabesmyia sp. 2 & - & 4,95 & Strixino \& Strixino, 2000 & & \\
\hline Clinotanypus sp. 1 & 0,44 & - & Chironomus sp. 1 & 5,33 & - \\
\hline Djamabatista sp. 1 & - & 1,10 & Chironomus sp. 2 & 0,67 & - \\
\hline Fittkauimyia sp. 1 & $\begin{array}{l}0,11 \\
2,22\end{array}$ & - & Chironomini Gên. A sp. 1 & - & 1,65 \\
\hline $\begin{array}{l}\text { Labrundinia } \text { sp. } 1 \\
\text { Labrundinia } \text { sp. } 2\end{array}$ & $\begin{array}{l}2,22 \\
0,67\end{array}$ & $\begin{array}{l}- \\
-\end{array}$ & Chironomini Gên. A sp. 2 & - & 0,27 \\
\hline Labrundinia sp. 3 & - & 3,57 & Chironomini Gên.B sp. 1 & - & 0,82 \\
\hline Larsia sp. 1 & 4,33 & 2,75 & Cladopelma forcipis (Rempel, & 4,11 & - \\
\hline Larsia $\mathrm{sp} .2$ & 0,11 & - & 1939) & & \\
\hline Monopelopia caraguata & - & 0,27 & Cryptochironomus sp. 1 & 0,11 & - \\
\hline Mendes, Marcondes \& & & & Endotribelos sp. 1 & - & 8,79 \\
\hline Pinho, 2003 & & & Endotribelos sp. 2 & - & 3,30 \\
\hline Pentaneura sp. 1 & 0,22 & - & Endotribelos sp. 3 & - & 1,10 \\
\hline Pentaneurini sp. 1 & 0,22 & 2,75 & Goeldichironomus neopictus & 0,11 & - \\
\hline cf. Trissopelopia sp. 1 & - & 0,55 & Trivinho-Strixino\&Strixino, 1998 & & 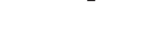 \\
\hline $\begin{array}{l}\text { Tanytarsini } \\
\text { Caladomyia } \mathrm{sp} .1\end{array}$ & - & 35,16 & Harnischia sp. 1 (complexo) & 4,22 & - \\
\hline Nandeva sp. 1 & - & 0,27 & Oukuriella sp.1 & - & 1,65 \\
\hline Rheotanytarsus sp. 1 & 0,11 & - & Parachironomus supparilis & 5,11 & - \\
\hline Rheotanytarsus sp. 2 & 4,33 & - & (Edwards, 1931) & & \\
\hline Stempellinella sp. 1 & - & 0,27 & cf. Pelomus sp. 1 & - & 1,92 \\
\hline Tanytarsini sp. 1 & 8,00 & - & Polypedilum sp. 1 & 0,33 & - \\
\hline Tanytarsini sp. 2 & 10,11 & - & Polypedilum sp. 2 & - & 1,65 \\
\hline Tanytarsini sp. 3 & 2,00 & - & Polypedilum sp. 3 & - & 0,55 \\
\hline Tanytarsini sp. 4 & 5,78 & - & Stenochironomus sp. 1 & - & 9,07 \\
\hline $\begin{array}{l}\text { Tanytarsini sp. } 5 \\
\text { Tanytarsini sp. } 6\end{array}$ & 4,44 & 5,49 & Xestochironomus sp. 1 & - & 0,55 \\
\hline Tanytarsini sp. 7 & - & $\begin{array}{l}5,49 \\
1,10\end{array}$ & Xestochironomus sp. 2 & - & 0,82 \\
\hline Tanytarsini sp. 8 & - & 0,27 & Xestochironomus sp. 3 & - & 2,47 \\
\hline Tanytarsini sp. 9 & - & 0,27 & Ortocladiinae & & \\
\hline Tanytarsini sp. 10 & - & 0,27 & Corynoneura sp. 1 & 0,11 & - \\
\hline Tanytarsini sp. 11 & - & 0,27 & Cricotopus sp. 1 & 15,56 & 0,55 \\
\hline Tanytarsini sp. 12 & - & 0,27 & Nanocladius sp. 1 & 0,44 & 0,27 \\
\hline Tanytarsini sp. 13 & 0,44 & - & Thienemanniella sp. 1 & 13,44 & 0,27 \\
\hline Virgatanytarsus sp. 1 & 2,67 & - & & & \\
\hline Virgatanytarsus sp. 2 & 0,89 & - & & & \\
\hline
\end{tabular}

rápida quando comparado com os procedimentos utilizados nos estudos de larvas e ainda fornece informação integrada da comunidade de Chironomidae do local, já que a coleta não é seletiva, pois todas as espécies emergem na superfície. Além disso, o método proporciona uma identificação mais acurada das espécies (Thienemann 1910; Coffman 1973). A estas vantagens ainda pode ser adicionada a natureza ética e não destrutiva do procedimento amostral.

Neste contexto, o objetivo deste estudo foi o de utilizar este método alternativo de coleta, ainda pouco usado no Brasil, para a caracterização e comparação da diversidade e riqueza de Chironomidae (Diptera) de dois córregos de baixa ordem na região central do Estado de São Paulo.

\section{MATERIAL E MÉTODOS}

Locais de coleta. As coletas foram realizadas em dois córregos localizados no campus da Universidade Federal de São Carlos (entre as coordenadas $21^{\circ} 58^{\prime}$ - 22 $2^{\circ} 00^{\prime}$ S e $47^{\circ} 51^{\prime}$ $47^{\circ} 52^{\prime}$ W) em São Carlos - SP e fazem parte da bacia do Tietê.
O córrego do Fazzari, de primeira ordem, possui pequenas dimensões (profundidade máxima de $0,60 \mathrm{~m}$, largura máxima de $1,50 \mathrm{~m})$, apresenta exuberante vegetação ripária no trecho analisado e situa-se numa área não urbanizada do campus com vegetação de cerrado stricto sensu (124,68 ha). O Rio do Monjolinho de maior porte (segunda ordem), profundidade máxima de $0,80 \mathrm{~m}$, largura máxima de $1,80 \mathrm{~m}$, percorre um trecho de aproximadamente $500 \mathrm{~m}$ na parte sul do campus da Universidade, a montante do reservatório homônimo e a área amostrada é desprovida de vegetação ripária.

Coleta e identificação do material. As coletas de exúvias, realizadas no período de estiagem (agosto/2001), foram feitas de duas maneiras, com redes de deriva tipo Brundin e redes de mão (puçá), ambas com malha de $250 \mu \mathrm{m}$. As redes de Brundin foram dispostas no canal dos córregos, em áreas de corredeira, para interceptar as exúvias em deriva, assim permanecendo por um período de 1 hora. As exúvias acumuladas nas margens das áreas de remanso ou em obstáculos como troncos e folhas, foram coletadas com a rede de mão, através do processo de varredura feita em uma extensão de 100 metros. Este 
Tabela II. Número de táxons (S), número de exúvias (N), índice de diversidade de Shannon-Wiener (H') e uniformidade de Pielou.(J) de Chironomidae no Córrego do Fazzari e Rio do Monjolinho.

\begin{tabular}{lcccc}
\hline & S & N & H' & J \\
\hline Córrego do Fazzari & 36 & 364 & 1,129 & 0,73 \\
Rio do Monjolinho & 31 & 900 & 1,210 & 0,81 \\
\hline Total & 61 & 1264 & & \\
\hline
\end{tabular}

procedimento foi adotado para padronizar o esforço amostral nos dois corpos d'água.

O material retido nas redes foi acondicionado em recipientes plásticos contendo água e transportado para o laboratório onde procedeu-se à triagem das exúvias, que foram preservadas em álcool $70 \%$. Exemplares dos diferentes morfótipos foram montados em lâminas com Euparal e examinados ao microscópio para identificação com o auxílio de bibliografia especializada (Wiederholm 1986; Coffman \& Ferrington 1996; Borkent 1984).

Análise dos dados. Após a identificação, os exemplares de cada sistema foram enumerados e analisados segundo a participação relativa de cada táxon. Para análise da diversidade de Chironomidae foi usado o índice de diversidade de Shannon-Wiener (H') e de equitabilidade de Pielou (J) (Zar 1999). Para a comparação entre os dois corpos d'água utilizouse, além da riqueza de espécies $(\mathrm{S})$ e do número de indivíduos $(\mathrm{N})$, o coeficiente de similaridade de Jaccard (Cj) (Magurran 1988) e porcentagem de similaridade (PSc) (Whittaker \& Fairbanks 1958 ). Para testar a diferença entre os valores do índice de diversidade de Shannon, utilizamos o teste t proposto por Hutcheson (Zar 1999).

\section{RESULTADOS EDISCUSSÃO}

Coletamos e identificamos 1264 exúvias distribuídas em 61 táxons, dos quais 31 (9 Tanypodinae, 10 Tanytarsini, 8 Chironomini e 4 Orthocladiinae) no trecho do Rio do Monjolinho e 36 (8 Tanypodinae, 10 Tanytarsini, 15 Chironomini e 3 Orthocladiinae) no Córrego do Fazzari (Tabela I). A subfamília Chironominae, representada pelas tribos Tanytarsini e Chironomini, apresentou participação mais expressiva nos dois sistemas, respectivamente com 39 e $20 \%$ da fauna no Rio do Monjolinho e 44 e 36\% no Córrego do Fazzari (Fig. 1). Os dois sistemas apresentaram baixa similaridade taxonômica com valores de coeficiente de Jaccard $=9,84 \%$ e Porcentagem de Similaridade $=7,13 \%$, indicando que os dois trechos analisados abrigam comunidades diferentes.

A maior riqueza observada no Córrego do Fazzari deve estar relacionada à presença da vegetação ripária bastante preservada no trecho analisado. Segundo Vannote et al. (1980) a vegetação nas margens dos rios tem um papel fundamental na estruturação do ambiente aquático; o aporte de material como folhas, troncos e frutos, contribui para o aumento da heterogeneidade ambiental e conseqüentemente maior disponibilidade de habitats a serem colonizados.

A presença de Beardius xylophylus (Trivinho-Strixino \&
Strixino 2000) e Stenochironomus sp.1, espécies associadas a madeira, é indicativa das características do substrato do Córrego do Fazzari que é rico em restos vegetais (troncos, galhos e folhas mortas). Outros autores como Trivinho-Strixino \& Strixino (1998) e Henriques-Oliveira et al. (2003) apontam a madeira como uma das principais fontes de alimento destes táxons. O mesmo pode ser dito a respeito dos outros morfótipos do complexo Stenochironomus (Xestochironomus sp.1, sp.2 e sp.3) (Borkent 1984). Outros táxons também relacionados ao aporte de restos vegetais da mata ciliar são aqueles do gênero Endotribelos, que no Córrego do Fazzari estiveram representados por três espécies, que contribuíram com aproximadamente $13 \%$ das exúvias coletadas no local. As larvas de Endotribelos têm sido encontradas com freqüência dentro de frutos e no interior de troncos em decomposição (Fabio Roque, comunicação pessoal). Enquanto no córrego do Fazzari muitos dos táxons encontrados estão relacionados com a presença da vegetação ripária, no trecho do Rio Monjolinho as macrófitas aquáticas, como Ludwigia e Miriophyllum, parecem ter sido os principais componentes determinantes da riqueza da comunidade de Chironomidae.

As diferenças no número de táxons e na participação das subfamílias e tribos de Chironomidae são apresentadas na figura 1. No Rio do Monjolinho, Orthocladiinae, com Cricotopus sp. 1 e Thienemanniella sp. 1, representou 29,56\% da fauna; Sanseverino \& Nessimian (2001) verificaram a presença de espécies do mesmo gênero em áreas de erosão em riachos da Mata Atlântica no Estado do Rio de Janeiro. Ao contrário, no Córrego do Fazzari, esta subfamília foi numérica e qualitativamente pouco expressiva (com 3 táxons e $1 \%$ das exúvias). Neste houve um maior contribuição da tribo Chironomini, mais freqüentemente relacionadas com áreas de depósito (Sanseverino \& Nessimian 2001; Roque \& TrivinhoStrixino 2001).

Um fato interessante refere-se à tribo Tanytarsini representada por 20 espécies, sendo 10 exclusivas de cada um dos sistemas (Tab. 1). Muito provavelmente os táxons de Tanytarsini do Rio do Monjolinho vivem associados às macrófitas aquáticas e/ou se beneficiando da maior velocidade da água do local, como é o caso de Rheotanytarsus cujas

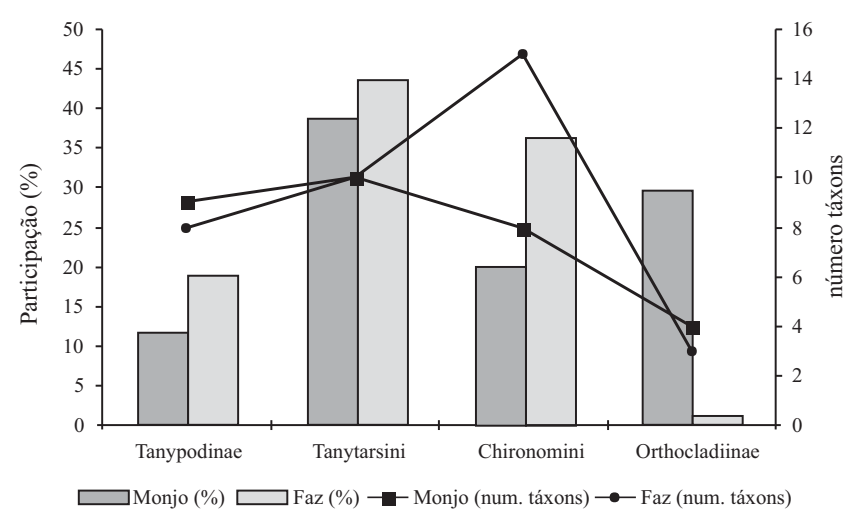

Fig. 1. Participação relativa (\%) e número de táxons no trecho do Rio do Monjolinho (Monjo) e no Córrego do Fazzari (Faz). 
espécies são tipicamente reobiontes (Wiederholm 1986).

O córrego do Fazzari mesmo com maior riqueza (36 morfoespécies), apresentou o índice de diversidade ( $\mathrm{H}^{\prime}=1,121$ nats/ind.) inferior $(\mathrm{t}=2,338, \mathrm{p}<0,05)$ ao obtido no Rio do Monjolinho ( $\mathrm{H}^{\prime}=1,210$ nats/ind.) (Tabela II). A equitabilidade $(\mathrm{J}=0,73)$ obtida no córrego do Fazzari, menor em relação à do Monjolinho $(\mathrm{J}=0,81)$, explica, em parte, o fato de um ambiente com maior número de espécies possuir o menor índice de diversidade. Esta baixa uniformidade obtida para a fauna do córrego do Fazzari tem como causa principal a elevada participação de Caladomyia sp. 1, táxon que sozinho contribuiu com $35,16 \%$ do total de exúvias (Tabela I).

Comparando nossos resultados podemos concluir que o número de espécies obtido através deste método (coleta de exúvias) foi muito superior daqueles observados por outros autores trabalhando apenas com as larvas. Sanseverino \& Nessimian (2001) identificaram 39 táxons em 11 córregos de baixa ordem na Mata Atlântica do Rio de Janeiro; Suriano \& Fonseca-Gessner (2004) coletaram 43 morfoespécies em 4 córregos de baixa ordem localizados no Parque Estadual de Campos do Jordão. Roque \& Trivinho-Strixino (2001) em estudo também realizado no Córrego do Fazzari listaram 30 morfoespécies de Chironomidae. Em todos estes trabalhos citados, as coletas foram realizadas em vários períodos e/ou em vários pontos, envolvendo portanto, um esforço amostral maior do que o empreendido no presente estudo, em que com apenas uma coleta foram registradas 61 morfoespécies em dois córregos.

Nossos resultados indicam que o método de coleta de exúvias é eficiente para a caracterização de sistemas lóticos de baixa ordem. O método mostrou-se também apropriado para levantamentos da biodiversidade de sistemas aquáticos, uma vez que as exúvias de pupas permitem melhor acuidade na identificação quando comparadas às larvas de Chironomidae.

Agradecimentos. Á Mateus Pepinelli pela ajuda no campo. Este trabalho contou com o apoio financeiro do PIBIC/UFSCar/CNPq.

\section{REFERÊNCIAS}

Borkent, A. 1984. The systematics and phylogeny of the Stenochironomus Complex (Xestochironomus, Harrisius and Stenochironomus) (Diptera: Chironomidae). Memoirs of the Entomological Society of Canada, 128: 269 p.

Coffman, W. P. 1973. Energy flow in a woodland stream ecosystem. The taxonomic composition and phenology of the Chironomidae as determined by the collection of pupal exuviae. Archiv für Hydrobiolie 71: 281-322.

Coffman, W. P. \& C. L. De La Rosa. 1998. Taxonomic Composition and Temporal Organization of Tropical and Temperate Species Assemblages of Lotic Chironomidae. Journal of The Kansas Entomological Society, 71: 388-406.

Coffman, W. P. \& L. C. Ferrington. 1996. Chironomidae, p. 635-754. In: R. W. Merrit \& K. W. Cummins (eds.). An introduction to aquatic insects of North America. Kendall-Hunt, Duduque, Iowa, USA, $862 \mathrm{p}$.

Hardwick, R. A.; P. D. Cooper; P. S. Cranston; C. L. Humphrey \& P. L. Dostine. 1995. Spatial and temporal distributions patterns of drifting pupal exuviae of Chironomidae (Diptera) in streams of tropical northern Australia. Freshwater Biology 34: 569-578.

Henriques-Oliveira, A. L.; L. F. M. Dorvillé \& J. L. Nessimian. 2003. Distribution of Chironomidae larvae fauna (Insecta: Diptera) on different substrates in a stream at Floresta da Tijuca, RJ, Brazil. Acta Limnologica Brasiliensia 15: $69-84$.

Humphrey, C. L.; K. A. Bishop \& V. M. Brown. 1990. Use of biological monitoring in assessment of effects of mining wastes on aquatic ecosystems of the Alligator Rivers Region, tropical northern Australia. Environmental Monitoring and Assessment 14: 139-181.

Humphrey, C. L. \& P. L. Dostine. 1994. Development of biological monitoring programs to detect mining waste impacts upon aquatic ecosystems of the Alligator Rivers Region, Northern Territory, Australia. Mitteilungen Internationale Vereinigung für theoretische und angewandte limnologie 24: 293-314.

Lehmann, J. 1981. Chironomidae (Diptera) aus FlieBgewassern Zentralafrikas. Teil II: Kivu-Gebiet, Oztzaire. Spixiana, Supplement 5: 1-85.

Magurran, A. E. 1988. Ecological diversity and measurement. Princeton, Princeton University Press, $179 \mathrm{p}$.

Mcgill, J. D.; R. S. Wilson \& A. M. Brake. 1979. The use of chironomid pupal exuviae in the surveillance of sewage pollution within a drainage system. Water Research 13: 887-894.

Pinder, L. C. V. 1986. The pupae of Chironomidae (Diptera) of the Holartic region- Introduction, p. 7-10. In: T. Wiederholm (ed.). Chironomidae of the Holartic region: Keys and diagnoses. Part 2. Pupae. Entomol. Scand. Suppl., 19.

Roque, F. O. \& S. Trivinho-Strixino. 2001. Benthic macroinvertebrates in mesohabitats of different spacial dimensions in a first order stream (São Carlos-SP). Acta Limnologica Brasiliensia 13: 69-77.

Ruse, L. P. 2000. A simple key to water quality based on chironomid pupal exuviae, p. 405-413. In: O. Hoffrichter (ed.). Late 20 ${ }^{\text {th }}$ Century Research on Chironomidae: an Anthology from $13^{\text {th }}$ International Symposium on Chironomidae. Shaker Verlag, Germany, $661 \mathrm{p}$.

Sanseverino, A. M. \& J. L. Nessimian. 2001. Hábitats de larvas de Chironomidae (Insecta, Diptera) em riachos de Mata Atlântica no Estado do Rio de Janeiro. Acta Limnologica Brasiliensia 13: 29-38.

Stur, E. U. Nolte \& E. J. Fittkau. 2000. Chironomids from a surfacedrift in a intermittent stream in tropical Brazil, p. 425-432. In: O. Hoffrichter (ed.). Late $\mathbf{2 0}^{\text {th }}$ Century Research on Chironomidae: an Anthology from the $13^{\text {th }}$ International Symposium on Chironomidae. Shaker Verlag, Germany, $661 \mathrm{p}$.

Suriano, M. T. \& A. A. Fonseca-Gessner. 2004. Chironomidae (Diptera) Larvae in streams of Parque Estudual de Campos do Jordão, São Paulo state, Brazil. Acta Limnologica Brasiliensia 16: 129-136.

Thienemann, A. 1910. Das Sammeln von Puppenhauten der Chironomiden. Eine bitte um Mitarbeit. Archiv für Hydrobiolie 6: $213-214$

Trivinho-Strixino, S. \& G. Strixino. 1998. Chironomidae (Diptera) associados a troncos de árvores submersos. Revista Brasileira de Entomologia 41: 173-178.

Vannote, R. L.; G. W. Minshall; K. W. Cummins; J. R. Sedell \& C. E. Cushing. 1980. The river continuum concept. Cannadian Journal of Fish Aquatic Science 37: 130-137.

Whittaker, R. H. \& C. W. Fairbanks. 1958. A study of plankton and copepod communities in the Columbia basin, Southeastern Washington. Ecology 39: 46-56.

Wiederholm, T. (ed.). 1986. Chironomidae of the Holartic region: Keys and diagnoses. Part 2. Pupae. Ent. Scand. Suppl., 28: 299$456 \mathrm{p}$.

Wilson, R. S. 1984. Monitoring the effect of sewage effluent on the Oxford canal using chironomid pupal exuviae. Journal of the Institution of Water and Environmental Management, 8: 171-182.

Wilson, R. S. \& J. D. Mcgill. 1979. The use of chironomid pupal exuviae for biological surveillance of water quality. Technical Memo $\mathbf{N}^{\circ}$. 18, Department of the Environment London. $20 \mathrm{p}$. Wilson, R. S. \& P. L. Bright. 1973. The use of chironomid pupal exuviae for characterizing streams. Freshwater Biology 3: 283-302.

Zar, J. H. 1999. Biostatistical analysis. Prentice-Hall, Upper Saddle River, New Jersey. 662 p.

Recebido em 21.IX.2004; aceito em 01.VIII.2005 